\title{
Detection of thyroid nodules by routine ultrasound
}

\author{
Adina GHEMIGIAN ${ }^{1,3}$, Mara CARSOTE ${ }^{1,3}$, Eugenia PETROVA ${ }^{1,3}$, Ana VALEA $^{2,4}$, \\ Nicoleta DUMITRU ${ }^{3}$, Andra COCOLOS 3 \\ 1"Carol Davila" University of Medicine and Pharmacy, Bucharest, Romania \\ 2"Iuliu Hatieganu" University of Medicine and Pharmacy, Cluj-Napoca, Romania

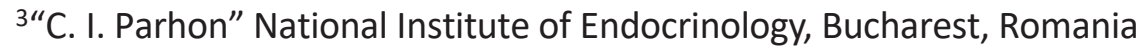 \\ ${ }^{4}$ Clinical County Hospital, Cluj-Napoca, Romania
}

\begin{abstract}
Ultrasound is an extremely useful tool of thyroid investigation showing different aspects from previously unknown nodules to high-risk lesions requiring immediate surgery. We aim to introduce a pictorial assay of a series cases from two tertiary Romanian centres of endocrinology underling different scenarios from detection to management. All female patients associated normal thyroid function and lack thyroid antibodies while thyroid ultrasound proved essential in detection and follow-up the thyroid condition. Case 1 is a 49-year old subject admitted after an episode of palpitations and detected with a left thyroid nodule of 4.9 by 2.7 by $4.9 \mathrm{~cm}$ with inhomogeneous pattern and relatively regular shape requiring surgery. Case 2 is a 63-year old patient accusing dizziness and requiring Doppler ultrasound for carotid arteries that incidentally pointed a thyroid nodule. Ultrasound confirmed a right lobe nodule of 1.1 by 0.6 by $1 \mathrm{~cm}$ in association with another small thyroid nodule of 0.8 by 0.6 by $0.6 \mathrm{~cm}$ having egg shell peripheral calcifications. Fine needle aspiration was indicated and follicular aspects were identified. Case 3 is a 56-year old female admitted for menopausal osteopenia and routine ultrasound found a hypo-echoic nodule of $2.5 \mathrm{~cm}$ at right lobe. Case 4 is a 67-year female who was actually post-operatory confirmed with $p$ T3aN1M0 papillary cancer starting from a first ultrasound evaluation of the thyroid. Case 5 is a 23-year old patient with a nodule detected by ultrasound three years ago and currently appreciated with a considerable increase even BETHESDA 2 was found initially through fine needle aspiration.

Conclusion. As pointed by present series of cases, thyroid ultrasound represents an essential method in the hands of endocrinologists.
\end{abstract}

Keywords: thyroid, ultrasound, nodule

\author{
Abbreviations \\ $\mathrm{cm}=$ centimetres \\ TSH $=$ Thyroid Stimulating Hormone
}

\section{INTRODUCTION}

Thyroid nodules represent a very frequent pathology in daily endocrine practice; their detection starts from self palpation, compressive symptoms, associated-thyroid mal-function or as an incidental finding during routine ultra- sound etc. (1-3). Also, more than half of patients with prior malignancies have an accidental thyroid nodule detected during computer tomography or magnetic resonance imagery performed for staging of the disease in order to establish the adequate management (4-6). Less than $10 \%$ of these cases actually underline a 
thyroid cancer and majority of them are not connected with primary site of the neoplasia since thyroid is not a typical region of metastasis (7-10).

\section{AIM}

Our purpose is to introduce five different cases associating different scenarios of diagnosis and presentation to whom routine ultrasound detected thyroid nodules larger than 1 centimetre $(\mathrm{cm})$, in the absence of thyroid function or autoimmunity anomalies.

\section{MATERIAL AND METHODS}

This is a cases series. The patients signed informed consent while they were evaluated at two different tertiary centres of endocrinology from Romania. The thyroid hormonal panel and ultrasound profile is provided, as well as most important aspects of medical background and accuses at presentation. This is a pictorial assay.

\section{RESULTS: CASES SERIES}

\section{Case 1}

This is 49-year old former smoker female who presented herself for an endocrine evaluation. She was first seen by her primary health physician accusing palpitations. Despite a normal electrocardiogram aspect and normal blood pressure, an endocrine assessment was considered useful. The family medical history is negative, the personal medical history includes cervical spondylosis; she has regular menses since the age of 12 years. At presentation, a left lobe thyroid nodule was detected by palpation and confirmed at ultrasound: the nodule displays the entire left thyroid nodule, having the diameters of 4.9 by 2.7 by $4.9 \mathrm{~cm}$ with inhomogeneous pattern and relatively regular shape (Fig. 1). The ultrasound background of thyroid is hypo-echoic, inhomogeneous; the right thyroid lobe is of 4 by 1.8 by $1.5 \mathrm{~cm}$ and it hosts a small nodule of 0.4 by $0.5 \mathrm{~cm}$. The specific thyroid blood assays were within normal limits (Table 1 ).

TABLE 1. Blood assays for thyroid profile in five cases with thyroid nodules at ultrasound

\begin{tabular}{|c|c|c|c|c|c|}
\hline Patient & Age (years) & TSH & FT4 & Calcitonin & TPOAb \\
\hline $\mathbf{1}$ & 47 & 1 & 10 & 1 & 10 \\
\hline $\mathbf{2}$ & 63 & 1.6 & 13.1 & 1 & 10 \\
\hline $\mathbf{3}$ & 56 & 1.4 & 16 & 1 & 10 \\
\hline $\mathbf{4}$ & 67 & 1.4 & 14 & 1.99 & 10 \\
\hline $\mathbf{5}$ & 23 & 3 & 13 & 1 & 10 \\
\hline Normal levels & & $0.5-4.5$ & $10.3-24.4$ & $1-4.8$ & $0-35$ \\
\hline Units & & $\mu \mathrm{UI} / \mathrm{mL}$ & $\mathrm{pmol} / \mathrm{L}$ & $\mathrm{pg} / \mathrm{mL}$ & $\mathrm{UI} / \mathrm{mL}$ \\
\hline
\end{tabular}

TSH = Thyroid Stimulating Hormone; FT4 = Free levothyroxine; TPOAb = anti-thyroperoxidase antibodies
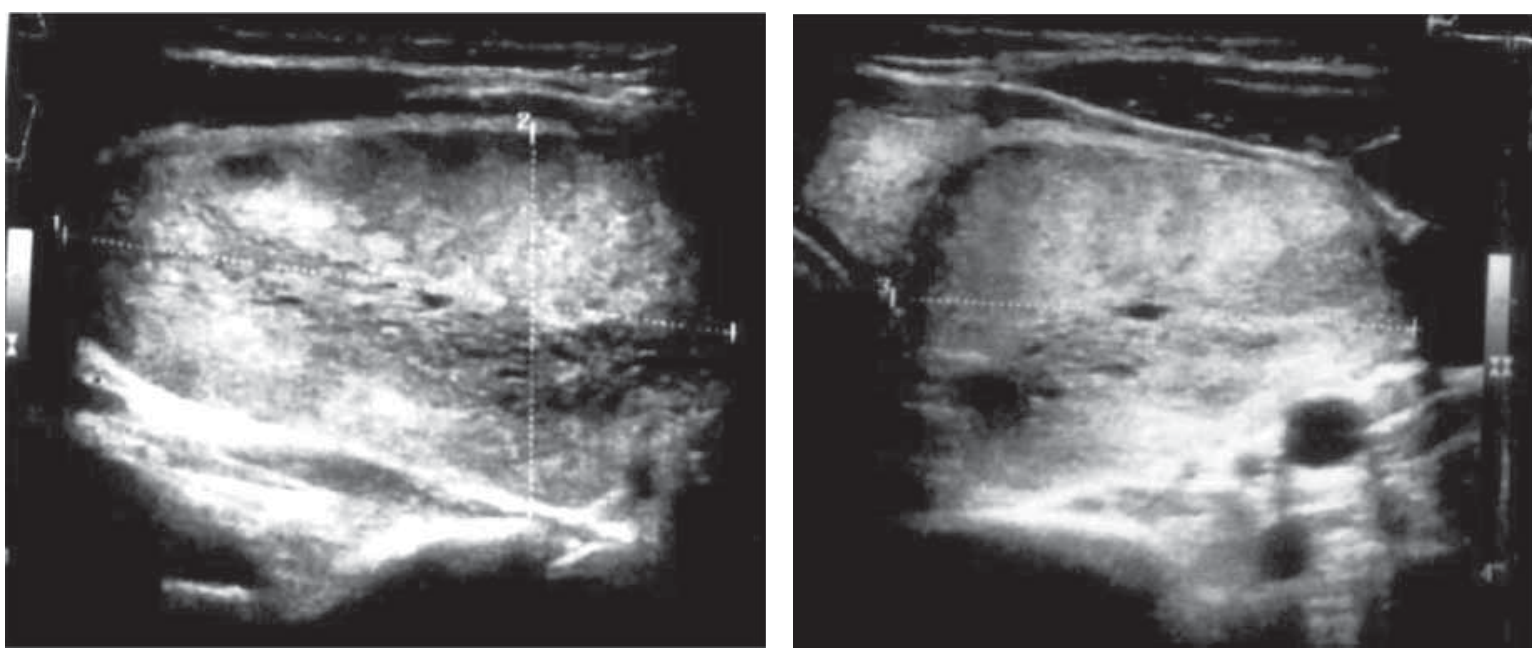

FIGURE 1. Thyroid ultrasound of 49-year old female showing micro-polynodular goitre with dominant left nodule 
Due to large size, the patient was referred to surgery which she is currently delaying.

\section{Case 2}

A 63-year old woman had a short episode of dizziness; neurologic exam identified a peripheral vestibular syndrome. A Doppler ultrasound exam of carotid arteries was necessary. During examination, although no specific pathologic condition was identified, a thyroid nodule was identified and the patient was referred for further evaluation. The subject is a formed heavy smoker for more than 30 years. The family medical history includes father with arterial hypertension. Personal medical references include: high blood pressure, dyslipidemia, anxious syndrome, essential tremor. The patient is normal weighted and reached menopause at age of 53 years. Thyroid blood assays were normal (Table 1). Thyroid ultrasound showed a right lobe with a nodule of 1.1 by 0.6 by $1 \mathrm{~cm}$ in association with another small thyroid nodule of 0.8 by 0.6 by $0.6 \mathrm{~cm}$ having egg shell peripheral calcifications (Fig. 2). The diameters of the two lobes were within normal limits: right of 3.4 by 1.6 by 1.6 $\mathrm{cm}$, left of 3.4 by 1.6 by $1.5 \mathrm{~cm}$. Fine needle aspiration was indicated and follicular aspects were identified.

\section{Case 3}

A 56-yeard old non-smoker female patient known with osteopenia was admitted for evaluation of this prior diagnosis. She has 14 years since menopause and previous diagnosis of polyrthorsis and recently a hypovitaminosis D (25-hydroxyvitamin D of $23 \mathrm{ng} / \mathrm{mL}$, optimal lev- els above $30 \mathrm{ng} / \mathrm{mL}$ ) was detected. A routine thyroid ultrasound scan was also done and introduced a hypo-echoic pattern, with normal size (of 3.1 by 1 by $1 \mathrm{~cm}$ on the right and 3.8 by 1.5 by $2.2 \mathrm{~cm}$ on the left) with a hypo-echoic, inhomogeneous right lobe nodule of 2.5 by 1.4 by $1.9 \mathrm{~cm}$ within transonic areas at interior in association with normal thyroid function and lack of positive autoimmunity (Fig. 3, Table 1). The patient refused fine needle aspiration and she was followed-up by thyroid ultrasound with status quo aspect after first three months since detection.

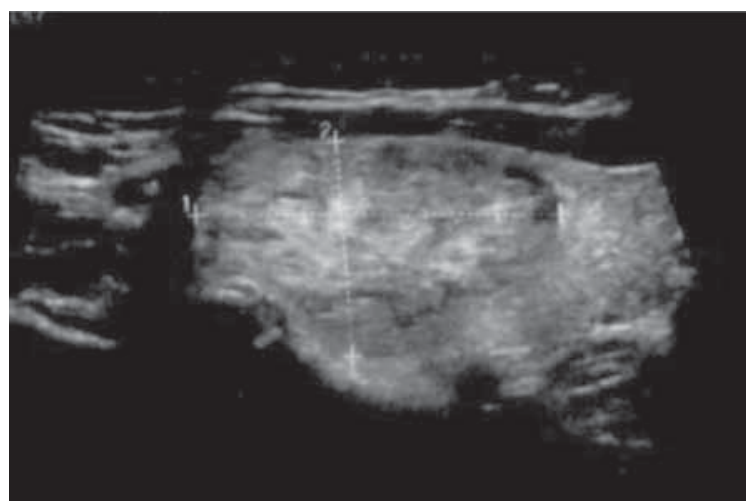

FIGURE 3. Thyroid ultrasound of 56-year old female revealing inhomogeneous right lobe nodule of 2.5 by 1.4 by $1.9 \mathrm{~cm}$

\section{Case 4}

This is 67-year old female who recently came to our attention for an ultrasound check-up of the cervical area after she observed an anterior cervical lump growing for the last several months. On admission, clinical evaluation detected an enlarged poly-nodular goitre with
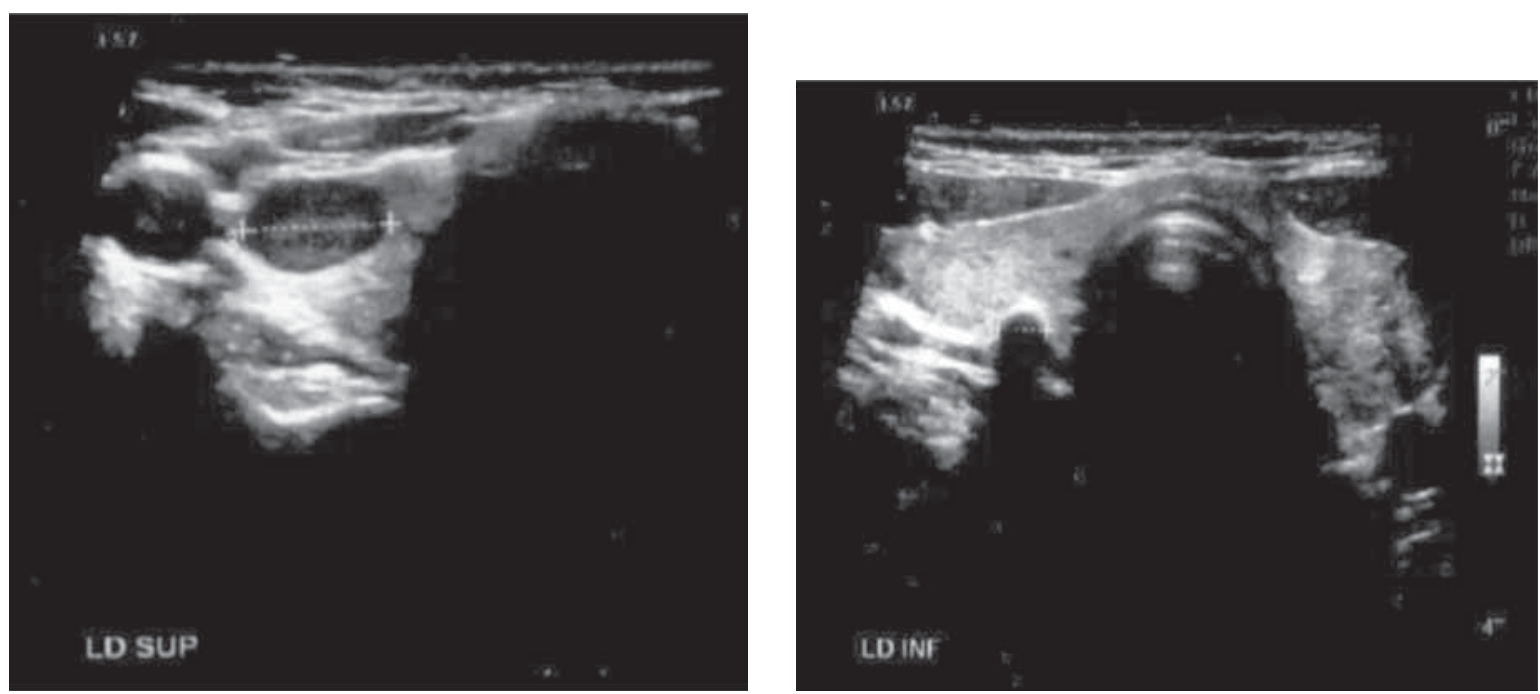

FIGURE 2. Thyroid ultrasound of 63-year old female pointing a right thyroid nodule of 1.1 by 0.6 by $1 \mathrm{~cm}$ (right) in association with another small thyroid nodule of 0.8 by 0.6 by $0.6 \mathrm{~cm}$ having egg shell peripheral calcifications (left) 

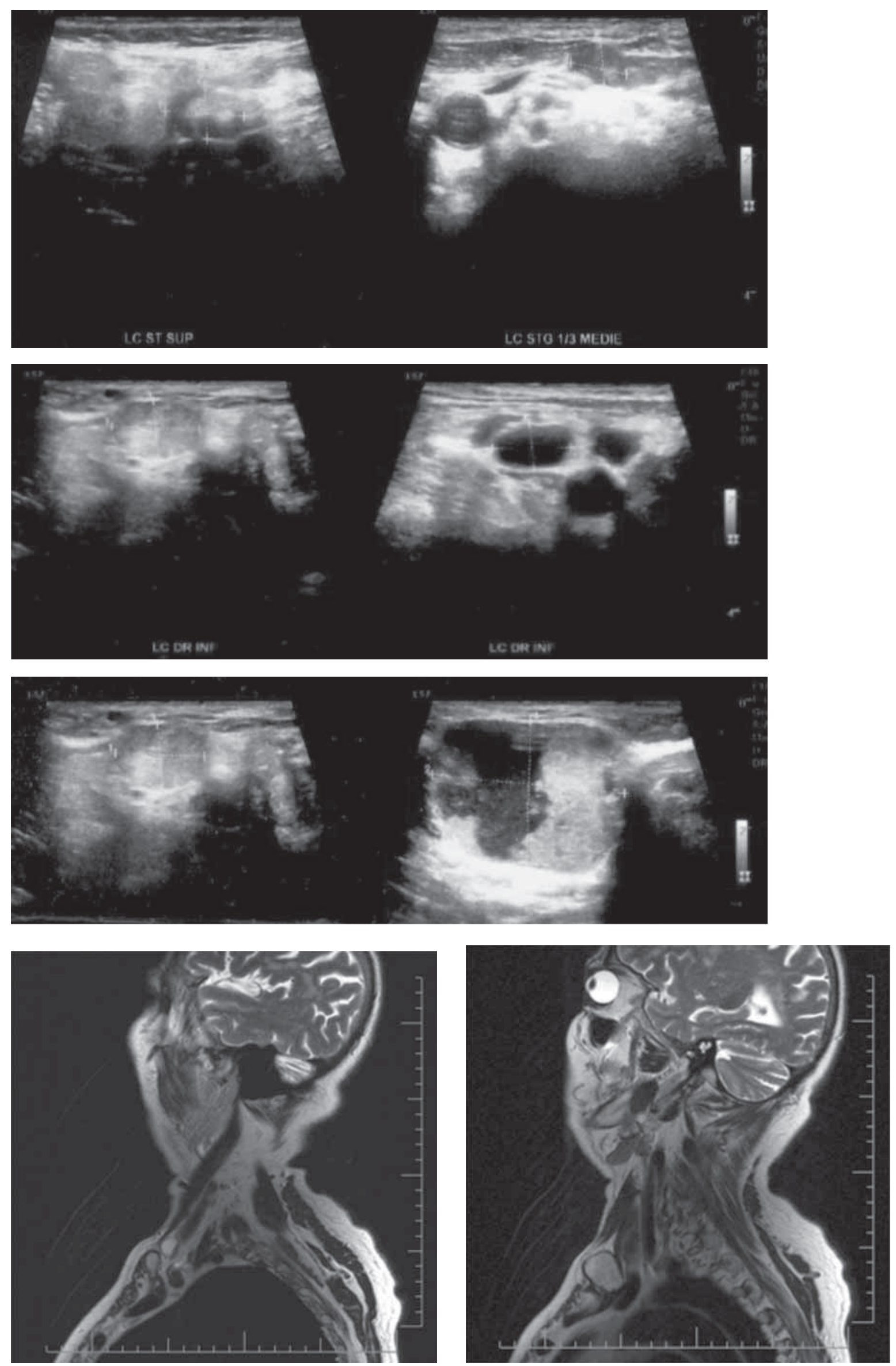

FIGURE 4. Thyroid ultrasound (first three lines), respective magnetic resonance imagery (last line) of 67-year old female identifying large multinodular goitre and cervical lymph nodes enlargement ( $p T 3 a N 1 M 0$ papillary thyroid cancer) 
multiple latero-cervical lymph nodes. She associated therapy for arterial hypertension, type 2 diabetes mellitus, and chronic cardiac ischemia. While no anomaly of thyroid function or autoimmune profile was detected, ultrasound indicated a large gland with a right lobe nodule of 3.4 by 2.9 by $2.8 \mathrm{~cm}$, having irregular shape and some calcifications spots inside while numerous lymph nodes were detected on both sides at anterior-lateral cervical region of more than $3 \mathrm{~cm}$ maximum diameter for the largest ones, an aspect which was confirmed after magnetic resonance imagery (Fig. 4, Table 1). Total thyroidectomy with lymph nodes dissection was immediately performed and confirmed a papillary thyroid cancer (pT3aN1M0). Vascular embolus from the primary site wad found during resection. Moreover, 7 out of 10 metastasis at the level of lymph nodes and, also, 10 out of 14 lymph nodes within surrounding adipose tissue were confirmed. Radioiodine therapy was added followed by suppression levothyroxine therapy.

\section{Case 5}

A 23-year old patient was accidently detected with a thyroid nodule three years ago by a routine ultrasound. Medical history of the family includes: mammary breast cancer (maternal grandmother) and type 2 diabetes mellitus (mother). Her personal medical history is irrelevant. At the moment of first diagnosis, typically follicular aspects (BETHESDA 2) were identified. No therapy was introduced for the following period of time. She remained asymptomatic until recently when intermittent breathing difficul- ties are described. The most recent assessment showed normal TSH (Thyroid Stimulating Hormone). Thyroid ultrasound showed a right macro-nodule of 3.5 by 2.9 by $1.55 \mathrm{~cm}$ which is $200 \%$ larger than initially and causes a mild left deviation of the trachea. The lymph nodes from cervical level are smaller than $1 \mathrm{~cm}$ and display nonspecific pattern. The patient was informed of the risks and referred to surgery.

\section{DISCUSSION}

This is a case series of five reports underlying cases where thyroid ultrasound was the key of detecting and further managing the thyroid condition. All the patients had normal TSH and lack positive autoimmunity profile although hypo/hyperthyroidism may associate dominant thyroid nodules but, frequently, the disturbances caused by lack or excess of thyroid hormones bring the patient to an evaluation rather than anatomical gland changes (11-15). The first two cases actually were sent from different medical specialities as neurology or cardiology while case number 3 was under endocrine revision for bone profile which is typically for menopause (16-18). Some of cases included as management fine needle aspiration which is a critical tool for obtaining direct information regarding the nodules in the absence of surgery and subsequent histological profile (19). Differential diagnosis of thyroid cancers as papillary or medullar is achievable through this method before thyroidectomy (20-22). The fourth case has actually the complete assays including post-operatory
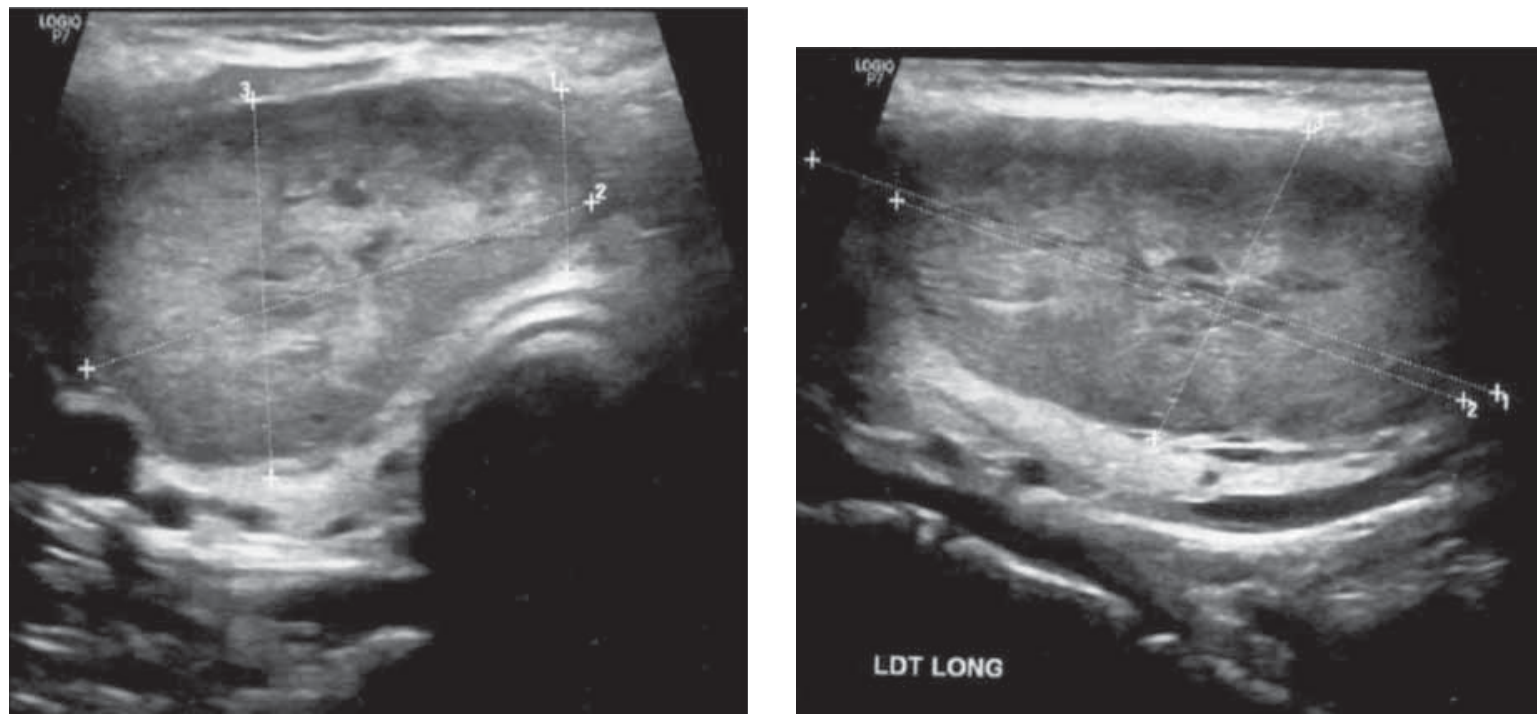

FIGURE 5. Thyroid ultrasound of 67-year old female highlighting a right macro-nodule of 3.5 by 2.9 by $1.55 \mathrm{~cm}$ 
pathological profile and it includes a very severe prognosis; further on, thyroid ultrasound is useful for routine follow-up as well as blood thyroglobuline and thyroid scintigrame (23-25). The fifth case is actually known with thyroid pathology and ultrasound proved useful for follow-up as well as for the first evaluation as seen in patients with prior thyroid or non-thyroid conditions $(26,27)$.

\section{CONCLUSION}

As pointed by present series of cases, thyroid ultrasound is an essential tool in the hands of endocrinologists.

\section{Acknowledgments}

We thank the medical teams that referred the patients to our departments.

Conflict of interest: none declared
Financial support: none declared

\section{REFERENCES}

1. Russ G., Bonnema S.J., Erdogan M.F. et al. European Thyroid Association Guidelines for Ultrasound Malignancy Risk Stratification of Thyroid Nodules in Adults: The EU-TIRADS. Eur Thyroid J. 2017; 6(5):225-237.

2. Dumitru N., Ghemigian A., Carsote M. et al. Thyroid nodules after initial evaluation by primary health care practitioners: an ultrasound pictorial essay. Archives of the Balkan Medical Union. 2016; 51(3):434-438.

3. Gheorghisan-Galateanu A.A., Carsote M., Valea A. Incientaloma: from general practice to specific endocrine frame. J Pak Med Assoc. 2017 ;67(6):917-922.

4. Tamhane S., Gharib H. Thyroid nodule update on diagnosis and management. Clin Diabetes Endocrinol. 2016;2:17.

5. Valea A., Ghervan C., Morar A. et al. Hashimoto's thyroiditis and breast cancer: coincidence or correlation?. Archives of Balkan Medical Union. 2016; 51(1):129-132.

6. Carsote M., Ghemigian A., Albu S.E. et al. Thryoid assessment and prostate cancer- related iatrogenic androgen deprivation. Archives of Balkan Medical Union. 2016; 51(1):124-128.

7. Farrá J.C., Picado O., Liu S. et al. Clinically significant cancer rates in incidentally discovered thyroid nodules by routine imaging. J Surg Res. 2017; 219:341-346.

8. Poiana C., Carsote M., Chirita C. et al. Thyroid nodules in patients with neuroendocrine tumors-two cases report, Timisoara Medical Journal, 2009; 59(4):371-374.

9. Poiana C., Carsote M., Ardeleanu C. et al. The value of the immunohistochemistry in a case of gastric neuroendocrine tumor and thyroid metastasis, Rom J Morphol Embryol, 2011; 52(1):187-192.

10. Carsote M., Valea A., Dumitru N. et al. Metastases in daily endocrine practice. Archives of Balkan Medical Union. 2016; 51(4):476-480.

11. Valea A., Albu S.E., Petrescu R., Carsote M. Chronic thyroiditis and differentiated thyroid cancer: from overlapping high prevalence to a shift on multifocal intra-thyroid papillary thyroid carcinoma. Journal of Surgical Sciences. 2017; 4(1):38-45.

12. Albu S.E., Carsote M., Terzea D. et al. Thyroid autoimmune disease: between hypothyroidism and hyperthyroidism. Archives of Balkan Medical Union. 2016; 51(4):481-485.

13. Gheorghisan-Galateanu A.A., Carsote M., Terzea D. et al. Premature ovarian failure and thyroid anomalies in patients with autoimmune disturbances. Gineco.eu Journal, 2015; 11(40):53-55.

14. Valea A., Carsote M., Morar A. et al. High cardio-metabolic risk parameters: a cross-sectional study in 80 women and men diagnosed with chronic thyroiditis (an age decades analysis). Current Health Sciences Journal. 2015; 41(Suppl. 1):101-104.

15. Paun D.L., Petris R., Carsote M. et al. Particular aspects in autoimmune thyroid diseases. Practica Medicala. 2013; 3(31):173-177.

16. Ghemigian A., Carsote M., Dumitru N. et al. The bone profile after surgery for diferentiated thryoid carcinoma. Current Health Sciences Journal. 2016; 42(S5):39-42.

17. Carsote M., Radu O., Dumitrascu A. et al. Bone and menopause: threshold of intervention. Romanian Medical Journal (Revista Medicala Romana). 2016; LXIII(3):233-236

18. Carsote M., Albu S.E., lorgulescu R. et al. From vasomotor symptoms to solid and insular papillary thyroid cancer with oxyphil variant areas. Journal of Surgical Sciences. 2015; 2(2):87-91.

19. Zafon C., Díez J.J., Galofré J.C., Cooper D.S. Nodular Thyroid Disease and Thyroid Cancer in the Era of Precision Medicine. Eur Thyroid J. 2017; 6(2):65-74.

20. Valea A., Radu O., Morar A. et al. Synchronous medullar thyroid cancer and primary hyperparathyroidism on a female within the sixth decade of life with positive family history for type 2A MEN syndrome. Journal of Medical Practice (Practica Medicala). 2016; 4(47)-11:346-349.

21. Haugen B.R. 2015 American Thyroid Association Management Guidelines for Adult Patients with Thyroid Nodules and Differentiated Thyroid Cancer: What is new and what has changed? Cancer. 2017; 123(3):372-381.

22. Poiana C., Popescu A., Carsote M. et al. Medullary carcinoma of the thyroid- limited therapeutic options, Infomedica; 2008; 4(146):43-48.

23. Melany M., Chen S. Thyroid Cancer: Ultrasound Imaging and Fine-Needle Aspiration Biopsy. Endocrinol Metab Clin North Am. 2017; 46(3):691-711.

24. Vasiliu C., Carsote M., Valea A. et al. From benign lymphoid hyperplasia of the tongue base to iatrogenic hypothyroidism and severe menopausal osteoporosis. Ginecologia.ro. 2016; 13(3):58-60.

25. Paschou S.A. Vryonidou A., Goulis D.G. Thyroid nodules: A guide to assessment, treatment and follow-up. Maturitas. 2017; 96:1-9.

26. Valea A., Carsote M., Ghemigian A., Dumitrache C. Pituitary macroadenoma and thyroid autoimmunity: cross-sectional observations in adult population. Current Health Sciences Journal. 2016; 42(S5):50-53.

27. Kelley S. Endocrinology Update: Thyroid Disorders. FP Essent. 2016;451:11-16. 